Bio - grafía. Escritos sobre la Biología y su Enseñanza. ISSN 2027-1034

Edición Extraordinaria. p.p. 112 - 120

Memorias del IX Encuentro Nacional de Experiencias en Enseñanza de la Biología y la Educación Ambiental. IV Congreso Nacional de Investigación en Enseñanza de la Biología.

\title{
LA ESCUELA: UN ESCENARIO PARA LOGRAR COMPROMISO CON LO NUESTRO.
}

THE SCHOOL: ASTAGE TO ACHIEVE COMMITMENT WITH OUR ENVIRONMENT.

\author{
Vanessa Díaz \\ Daniel Salazar \\ Tatiana Lara \\ Nicolasa Díaz \\ Alexander Alfonso \\ Paula Salcedo \\ Rosa María Galindo ${ }^{1}$
}

\section{RESUMEN}

Esta ponencia pretende asumir la educación como una práctica política lo cual conlleva a un compromiso ético- político que implica el compartir social de vínculos y de responsabilidades respecto a: otros quienes están enmarcados en concepciones de mundo que responden a los sistemas ideológicos en los que subyacen y que determinan su carácter como individuos; y respecto a las implicaciones que crean las relaciones que se establecen con el territorio que se habita. Es así como a través de diversos proyectos heterogéneos desarrollados desde la propuesta metodológica de las ATAs en el espacio de economía azul de la Escuela Pedagógica Experimental; se evidencia cómo el aprendizaje de la biología está íntimamente relacionado con la posibilidad de trabajar en torno a la re-configuración de la relación entre lo humano y la naturaleza que implica no solo hacer-nos conscientes que somos naturaleza sino generar un diálogo entre lo local y lo global, entre el saber científico y el saber popular ,y entre la escuela y la comunidad de la que hace parte la institución escolar.

PALABRAS CLAVES: Compromiso político, aprendizaje biología, problemáticas reales y contexto local y global.

\section{SUMMARY}

Throughout this text, we intend to assume education as a political practice which leads to an ethical-political commitment that implies the social sharing of links and responsibilities regarding: others who are framed in conceptions of the world that respond to the ideological systems In which they grew up and which determine their

1 Escuela Pedagógica Experimental. vanessa.diaz@epe.edu.co, daniel.salazar@epe.edu.co, tatiana.lara@epe.edu.co,_nicolasa.diaz@epe.edu.co,_alexander.alfonso@epe.edu.co, paula.salcedo@epe.edu.co, rosa.galindo@epe.edu.co. 


\section{Bio - grafía. Escritos sobre la Biología y su Enseñanza. ISSN 2027-1034}

Edición Extraordinaria. p.p. 112 - 120

Memorias del IX Encuentro Nacional de Experiencias en Enseñanza de la Biología y la Educación Ambiental. IV Congreso Nacional de Investigación en Enseñanza de la Biología.

character as individuals; and moreover, according to the implications that create the relations that are established with the territory where they live. In this sense, the heterogeneous projects developed under the methodological proposal of the ATAs in the Blue Economy subject at the Escuela Pedagógica Experimental evidence how the learning of Biology is intimately related to the possibility of working around the reconfiguration of the relationship between the human and the nature. This implies not only making us aware that we are nature but it also generates a dialogue between the local and the global, between scientific knowledge and popular knowledge, and between the school and the community of which the school is part.

KEYWORDS: Political commitment, learning biology, real problems and local and global context.

\section{A MANERA DE PRESENTACIÓN}

La educación por sí misma no cambia el mundo, pero sin ella es imposible hacerlo. En consecuencia, el educador progresista debe tener un compromiso ético político por la construcción de un país más justo. El educador ve la historia como posibilidad no debe perder su capacidad de indignación, no puede ser indiferente ni neutral frente a las injusticias, la opresión, la discriminación y la explotación. (Torres A. 2012:p32)

Esta situación que nos plantea el maestro Alfonso Torres se hace evidente en los problemas asociados con la contaminación y degradación de los ecosistemas, el agotamiento de recursos, el crecimiento incontrolado de la población mundial, el uso de los alimentos para producir combustibles, los conflictos destructivos, la pérdida de diversidad biológica y cultural. Esta situación de emergencia planetaria aparece asociada a comportamientos individuales y colectivos orientados a la búsqueda de beneficios particulares y a corto plazo, sin atender a sus consecuencias para los demás o para las futuras generaciones.

La escuela no puede ser indiferente ante esta situación de crisis planetaria que nos invita a asumir un compromiso de manera que se aboquen situaciones cotidianas, locales y globales para lograr avanzar en una percepción sistémica de los problemas de nuestro tiempo. Desde aquí es posible fomentar actitudes y comportamientos favorables para el logro de un futuro sostenible. Se trata, en definitiva, de contribuir a formar ciudadanos conscientes tanto de la gravedad y del carácter global de los problemas, como de la necesidad de construir, recrear e inventar posibles rutas de trabajo de manera que se transforme la mirada en torno a la ciencia y a su compromiso con el contexto social-cultural y político que permita la toma de decisiones adecuadas.

Esta búsqueda requiere una reflexión permanente acerca de las realidades ambientales, políticas y económicas del presente, en donde se reconstruyen, formulan y legitiman nuevas miradas de una sociedad más humana. Entonces, pensar la educación como práctica política conlleva un compromiso ético-político que invita a plantear distintas búsquedas desde el pensamiento latinoamericano, modelando algunos de los elementos 


\section{Bio - grafía. Escritos sobre la Biología y su Enseñanza. ISSN 2027-1034}

\section{Edición Extraordinaria. p.p. 112 - 120}

Memorias del IX Encuentro Nacional de Experiencias en Enseñanza de la Biología y la

Educación Ambiental. IV Congreso Nacional de Investigación en Enseñanza de la Biología.

básicos de la participación política y de la construcción del saber que entreteje lo humano y la naturaleza, lo local y lo territorial en un mundo globalizado.

Desde allí la Escuela Pedagógica Experimental -EPE- ha configurado un espacio de trabajo colectivo entre maestros de diversas disciplinas, denominado Economía Azul, en donde desde la reflexión permanente, se ha planteado como derroteros: ¿Qué problemáticas ambientales identifican los estudiantes en la Escuela y fuera de ella? ¿Cómo constituir propuestas de trabajo que estén articuladas con el contexto? ¿Cómo construir colectivos de trabajo (estudiantes, maestros, sabedores de la comunidad) que asuman un compromiso ético-político? ¿Cómo se ha transformado la propuesta pedagógica de Economía Azul en la EPE?

Con este panorama, realizamos este ejercicio escritural que sistematiza la experiencia de la EPE, en donde se hace una apuesta buscando no sólo poder reflexionar sobre las implicaciones que tiene las nuevas alternativas de desarrollo "sostenible", las cuales requieren darse cuenta que cuanto más estudiamos los problemas ambientales de nuestro tiempo, más nos percatamos de que no pueden ser entendidos de manera aislada. Entonces, es necesario generar cambios de percepción para que se asuman de manera sistémica, pues existen fenómenos que están interconectados y son interdependientes. Esta manera de ver implica movilizar nuestro pensamiento, recrear nuevas formas de relación con el planeta y revisar nuestros valores para garantizar nuestra supervivencia.

\section{METODOLOGÍA}

Siendo coherentes con estas preocupaciones, los proyectos en la Escuela Pedagógica Experimental se desarrollan dentro de la propuesta de las ATAs, Actividad Totalidad Abiertas (Segura, 1995), que pretende una construcción cognitivo - afectiva entre el deseo del estudiante en la actividad y en el mantenimiento del interés por lo que se realiza en la clase, fundamentado por la existencia de un problema con el que se está comprometido, que no se ha resuelto pero que íntimamente se siente como posible de resolver. Esta propuesta metodológica permite que los problemas vivenciales de la cotidianidad de nuestro contexto se conviertan en el punto de partida de los proyectos a desarrollar; por tanto, lo importante para que se den dinámicas de aprendizaje es la construcción de ambientes educativos que generen un compromiso verdadero mediados por el hacer, la construcción de conocimiento y la transformación de las miradas del mundo.

Para que se logre una construcción holística del conocimiento, desde estas dinámicas de aprendizaje, es necesario reconocer diferentes saberes, no necesariamente producto de la educación formal, sino desde la experiencia; es así como en la EPE se tienen maestros campesinos, indígenas, líderes comunitarios, entre otros. Esta propuesta metodológica de reconocimiento de los saberes ha permitido, a lo largo de la experiencia en Economía Azul, contar con el aporte significativo de algunas de estas personas a las que se han denominado especialistas, en tanto son ellos los que poseen y han construido 


\section{Bio - grafía. Escritos sobre la Biología y su Enseñanza. ISSN 2027-1034}

Edición Extraordinaria. p.p. 112 - 120

Memorias del IX Encuentro Nacional de Experiencias en Enseñanza de la Biología y la

Educación Ambiental. IV Congreso Nacional de Investigación en Enseñanza de la Biología.

el conocimiento vivencial necesario para solucionar las diferentes problemáticas ambientales a las que nos vemos enfrentados en nuestra cotidianidad.

Las ATAs buscan cambiar la relación habitual maestro-estudiante-contenidos por otra en la que la actividad juega un papel especial, y así transformar el sistema y convertirlo en un sistema abierto. Tenemos entonces la tríada maestro - estudiante actividad; en ésta, la actividad se mantiene permanentemente interactuando con el contexto o entorno, dándole significado y pertinencia a la actividad. La actividad se constituye en el elemento determinante de la apertura del sistema en cuanto no se concibe como una estrategia para llegar a los contenidos, sino que por el contrario se transforma en el elemento de pertinencia que le da sentido a lo que se hace. Por ejemplo, cuando se emprende como problema de trabajo la recuperación de la quebrada que colinda con la Escuela, la cual ha sido contaminada y para ello se adelantan estudios fisicoquímicos del cuerpo de agua, también permitiendo ponerse en contacto con las autoridades pertinentes, formalizar vínculos con la comunidad que es afectada por la problemática, adelantar gestiones con alguna universidad para lograr asesorías sobre las diferentes aristas del asunto como la reforestación o los estudios de las aguas, entre otros.

En este caso nuestro problema es la quebrada contaminada y nuestra meta real es lograr descontaminarla. Que en este proceso existan muchos aprendizajes de diversas áreas es muy importante, pero las actividades no se plantean para aprender biología, química, ni silvicultura sino para recuperar la quebrada y lo que suceda con ella mantiene la atención de estudiantes y maestros en torno a una preocupación que existe fuera de la escuela y fuera de los contenidos previstos curricularmente.

Bajo esta perspectiva y al ser la Economía Azul un espacio no sólo para reflexionar sobre los problemas cotidianos, sino también para mirar la producción económica como una puesta que respete y esté en armonía con la naturaleza y sus procesos; se pretende en estas propuestas abordar ciertas problemáticas desde visiones amplias, complejas y productivas, que evidencien cambios significativos en los hábitos y en las dinámicas de los estudiantes, sus familias y la comunidad y especialmente cambios en la construcción de posturas políticas que cuestionen de manera crítica la realidad de nuestro país.

\section{RESULTADOS Y DISCUSIÓN -UNA HISTORIA QUE SE RE-CONFIGURA-²}

Los maestros de la Escuela Pedagógica Experimental consideramos que el conocimiento es posible, pues existen diversas maneras de aprender y es posible hacer de él algo útil, algo palpable que transforma y crea conciencia desde las distintas prácticas pedagógicas, las cuales nos permiten entender la realidad que vive el planeta y a la vez desarrollar nuestro compromiso en buscar alternativas a este modelo de

\footnotetext{
${ }^{2}$ Parte de estos resultados fueron expuestos en la ponencia: la versión pedagógica de la economía azul: una alternativa para la formación de maestros comprometidos con las problemáticas locales desde la educación ambiental y la construcción de paz. Escrita por las maestras Gloria Escobar y Vanessa Díaz para el evento de Educyt - Neiva 2016.
} 


\title{
Bio - grafía. Escritos sobre la Biología y su Enseñanza. ISSN 2027-1034
}

Edición Extraordinaria. p.p. 112 - 120

\author{
Memorias del IX Encuentro Nacional de Experiencias en Enseñanza de la Biología y la \\ Educación Ambiental. IV Congreso Nacional de Investigación en Enseñanza de la \\ Biología.
}

desarrollo devastador. Esta apuesta por la formación ética-política implica el compartir social de vínculos y de responsabilidades respecto a los otros, que están enmarcados en concepciones de mundo que responden a los sistemas ideológicos subyacentes y determinantes del carácter de los compromisos éticos y políticos de individuos como lo plantean Franco Frabboni y Franca Pinto:

Un paradigma ético -social comprometido con valorizar y respetar la pluralidad de los nuevos sujetos de derecho y de sus respectivas instancias: desde los derechos humanos hasta los derechos de la naturaleza, y esto es de los animales, de las plantas, del ecosistema, del todo... (2007p.84).

Es así que desde el año 2012 se consolida en la EPE la propuesta pedagógica de la Economía Azul, que retoma los planteamientos de Gunter Pauli, un emprendedor belga quien plantea maneras sostenibles de producción ecológica basado en la imitación de sistemas naturales, lo que nos permite replantearnos nuevas maneras de re-conectarnos con la naturaleza. Esta posibilidad de ofrecer alternativas innovadoras, rentables, sin residuos que afecten el ecosistema y comprometidas con el contexto, se ha convertido en un horizonte de posibilidades en nuestra Escuela para configurar diversos proyectos interdisciplinares, donde participan estudiantes de grado noveno a once que son acompañados por uno o varios maestros y en ocasiones por un especialista, un sabedor de la comunidad que nos enriquece de manera permanente. Esta apuesta busca comprender la manera cómo funciona la naturaleza y cómo se desarrollan los procesos que se gestan en su interior para que sea posible la vida.

La economía azul se basa en la regeneración, esto consiste en asegurar que los ecosistemas se mantengan en su trayectoria evolutiva de manera que podamos beneficiarnos del inagotable caudal de creatividad, adaptación y abundancia de la naturaleza (Pauli, 2010 p.21).

Desde esta perspectiva teórica, la Escuela empieza a retomar, reestructurar y valorizar muchas de las prácticas que se han venido desarrollando durante estos 40 años de existencia y que históricamente se han encaminado en el mismo sentido, por ejemplo el estudio de especies nativas como las lagartijas, la construcción del arboreto, el proyecto de mazacotes y menjurjes, la preparación de tintes naturales, discusiones sobre la seguridad alimentaria, etc.; y se inicia un proceso de estudio de los planteamientos teóricos de la Economía Azul dando surgimiento a la construcción de diversos proyectos liderados por maestros de diferentes áreas (sociales, matemáticas, idiomas, física, biología, educación de segunda infancia, música, artesanías, etc.), y comprometidos con las problemáticas locales reales. Es de notar que, a diferencia de otros espacios académicos, en el desarrollo de estos proyectos, la disciplina deja de ser el eje central de la clase; permitiendo la configuración de espacios en donde los conocimientos empíricos y ancestrales sumados a la experiencia pedagógica de cada docente se convirtieron en una estrategia que contempla la educación ambiental como un vehículo para la construcción de justicia y equidad.

Teniendo en cuenta la experiencia vivida en la EPE antes del 2012 y la lectura de los planteamientos del libro de Gunter Pauli, se decide adecuar el horario académico de la Escuela disponiendo dos horas semanales para hacer un estudio teórico de la propuesta 


\section{Bio - grafía. Escritos sobre la Biología y su Enseñanza. ISSN 2027-1034}

Edición Extraordinaria. p.p. 112 - 120

Memorias del IX Encuentro Nacional de Experiencias en Enseñanza de la Biología y la

Educación Ambiental. IV Congreso Nacional de Investigación en Enseñanza de la Biología.

de Pauli con los estudiantes; sin embargo, para iniciar el año 2013 se hace necesario hacer un reajuste bajando la intensidad horaria (1 hora) de las áreas de biología, inglés y química para proporcionarle 5 horas semanales a la franja llamada "Economía Azul" donde a partir de las discusiones e iniciativas que surgieron con los estudiantes en el año 2012 en los diferentes espacios, se plantean los siguientes proyectos: (Anexo 1).

Estas prácticas que hemos configurado como colectivo de maestros emergen desde la experiencia misma, donde hay posibilidad para cuestionar y pensarse como sujetos que propician escenarios de escucha, diálogo, construcción de saberes y posibilidades de vida, donde no hay límites para preguntar, para aprender, soñar e imaginar. Aunado a esto, tenemos que el espacio que da la Escuela para el aprendizaje, no sólo emerge desde un lugar y las relaciones de poder y orden que allí están presentes; más que eso, surge a partir de la construcción y tejido de saberes que se dan en torno al contexto, y así mismo, desde la problematización y análisis de los fenómenos que se considera pertinentes para abordar desde la escuela como lo plantea Masschelein, J. \& Simons, M:

Se acusa reiteradamente a la escuela de estar alejada del mundo. Que fracasa a la hora de abordar lo que es importante en la sociedad; que se ocupa de destrezas y conocimientos obsoletos y estériles; que los profesores se preocupan demasiado por minucias sin importancia y por la jerga académica. En respuesta a esas acusaciones, nosotros queremos argumentar que la profanación y la suspensión hacen posible abrir el mundo en la escuela y que en realidad es el propio mundo y no los talentos de los alumnos, o sus necesidades individuales de aprendizaje lo que se revela. $(2014$, p.20)

En este sentido, las transformaciones que se han dado en la Escuela con respecto al estudio e implementación pedagógica de la Economía Azul desde hace 6 años son muchas: 2012- Establecimiento de proyectos encaminados a abordar las problemáticas locales, 2013- dedicación de 5 horas semanales, trabajo conjunto con miembros de la comunidad que poseen tanto el saber empírico como tradicional y reconocimiento de experiencias cercanas, 2014- creación de proyectos productivos y trabajo con entidades tanto privadas como del gobierno 2015 - establecimiento de cinco líneas de trabajo (Energías alternativas, ¿ Quiénes somos?, ¿Y los residuos qué?, Nuestro territorio y Seguridad alimentaria) que si bien a lo largo de la historia en la Escuela han sido objeto de estudio, en este punto se convierten en el eje central de todos los proyectos que se adelantan desde las diferentes áreas y espacios tanto homogéneos como heterogéneos, 2016/2017 - enriquecimiento de los proyectos desde nuevas visiones que se trabajan en América Latina "El buen vivir, vivir bien"

\section{CONCLUSIONES}

En este proceso de reconstrucción de la memoria del proyecto de economía azul se logró visibilizar los saberes propios que se gestan desde la comunidad que al ser compartidos en nuestra escuela permiten re-conocer-nos como un tejido social en el mismo territorio a través de un diálogo de saberes y experiencias que apuntan a la 


\section{Bio - grafía. Escritos sobre la Biología y su Enseñanza. ISSN 2027-1034}

\section{Edición Extraordinaria. p.p. 112 - 120}

\section{Memorias del IX Encuentro Nacional de Experiencias en Enseñanza de la Biología y la \\ Educación Ambiental. IV Congreso Nacional de Investigación en Enseñanza de la Biología.}

formación de ciudadanos responsables consigo mismo, con los otros y con el entorno que los rodea.

Entonces, la posibilidad de lograr articulación de la escuela con el territorio es un intento por establecer un entramado de relaciones que busca vincular el aprendizaje escolar con la educación para la vida sin perder de vista que deben estar vinculadas con el contexto cultural de los niños y los jóvenes para que resulten valiosas y pertinentes. Es desde allí que pensar el territorio implica reflexionar sobre diferentes aspectos que atraviesan la vida y cotidianidad de todos sus habitantes; se trata entonces de un intento por develar aquellos puntos relacionales donde la vida, la política, la ciudadanía, el territorio y la participación nos atraviesan como seres humanos, no sólo en el marco de los discursos sino en el nivel de las decisiones y posibilidades de construcción de sí mismos como comunidad, territorio y región.

Sin duda alguna, en este transitar, hemos venido valorizando y reflexionando como comunidad educativa sobre la relación entre la memoria- la naturaleza y la vida en cada una de las acciones emprendidas; reflejo de esto, para este año (2017), dentro los proyectos adelantados encontramos: Cuidadores del tejido de la Vida, Recuperación del Arboreto y de la Quebrada Puente Piedra, energías alternativas, somos lo que comemos, espacios azules y amasijos arquitectónicos.

De esta manera, se intenta reconocer los roles que juega nuestra participación en la decisiones colectivas e individuales desde nuestras prácticas, las cuales configuran posibilidades de ser en lo local y lo global. Es así que nuestras búsquedas pedagógicas e investigativas se entrelazan con nuevas miradas acerca de nuestro presente y porvenir, sobre nuestro ser y deber ser; acercándonos al Buen vivir, Vivir bien y validando el pensamiento latinoamericano de una manera holística, valorando las raíces ancestrales que son presentadas en dos variables: El Buen vivir de la tradición ecuatoriana y el Vivir bien de la tradición boliviana, las cuales se cruzan, pues, tienen en común una búsqueda que ha unido las luchas de resistencia de nuestros pueblos originarios por darle lugar en su geografía y territorio como una necesidad de salvaguardar la naturaleza como patrimonio de la unidad del mundo. (Aguirre N. \& lbáñez A. 2013).

Para finalizar, esta es una Utopía en proceso de construcción que tiene que ver con otra forma de vida, con una serie de derechos y garantías sociales, económicas y ambientales que se rescatan de nuestros pueblos en la configuración de una vida armónica. En esencia, se trata de construir una economía solidaria, al tiempo que se recuperan varias soberanías como concepto central de la vida política del país como lo plantea el economista Acosta Espinosa (2009: 179). Para esto, es importante tener en cuenta que el fundamento de una nueva sociedad no es posible sin la comunidad soberana y autosuficiente. Esto requiere generar cambios de percepción frente a la relación consigo mismo, con la comunidad y con la naturaleza para asumir la vida de manera sistémica, esto es, poder reconocer que todos estamos interconectados y somos interdependientes. Esta manera de ver implica entonces, que la escuela apunte a movilizar nuestro pensamiento, a recrear nuevas formas de relación con el planeta y revisar nuestros valores de manera que permita garantizar nuestra supervivencia. 
Bio - grafía. Escritos sobre la Biología y su Enseñanza. ISSN 2027-1034

Edición Extraordinaria. p.p. $112-120$

Memorias del IX Encuentro Nacional de Experiencias en Enseñanza de la Biología y la

Educación Ambiental. IV Congreso Nacional de Investigación en Enseñanza de la Biología.

\section{FUENTES BIBLIOGRÁFICAS}

Acosta A.(2009). La maldición de la abundancia, Quito: Comité Ecuménico de proyectos.

Aguirre N. \& Ibáñez A. (2013). Una utopía en proceso de construcción. Colección Primeros pasos. Ediciones desde abajo. Bogotá, Colombia.

Frabboni F. \& Pinto F. (2007). Introducción a la Pedagogía General. México: Editores Siglo Veintiuno.

Masschelein, J. \& Simons, M (2014) Defensa de la escuela: Una cuestión Pública. Buenos Aires, Argentina. Editorial Buenos Aires, Miño \& Dávila.

Pauli G. (2010). La Economía Azul. México: Tusquets Editores.

Segura, d. y. (1995). Vivencias de Conocimiento y Cambio Cultural. Bogotá D.C.: Escuela Pedagógica Experimental.

Torres A. (2012). La Educación popular. Trayectoria y actualidad. Universidad Bolivariana de Venezuela. Editorial el Buho. 
Bio - grafía. Escritos sobre la Biología y su Enseñanza. ISSN 2027-1034

Edición Extraordinaria. p.p. 112 - 120

Memorias del IX Encuentro Nacional de Experiencias en Enseñanza de la Biología y la Educación Ambiental. IV Congreso Nacional de Investigación en Enseñanza de la Biología.

\section{ANEXO 1}

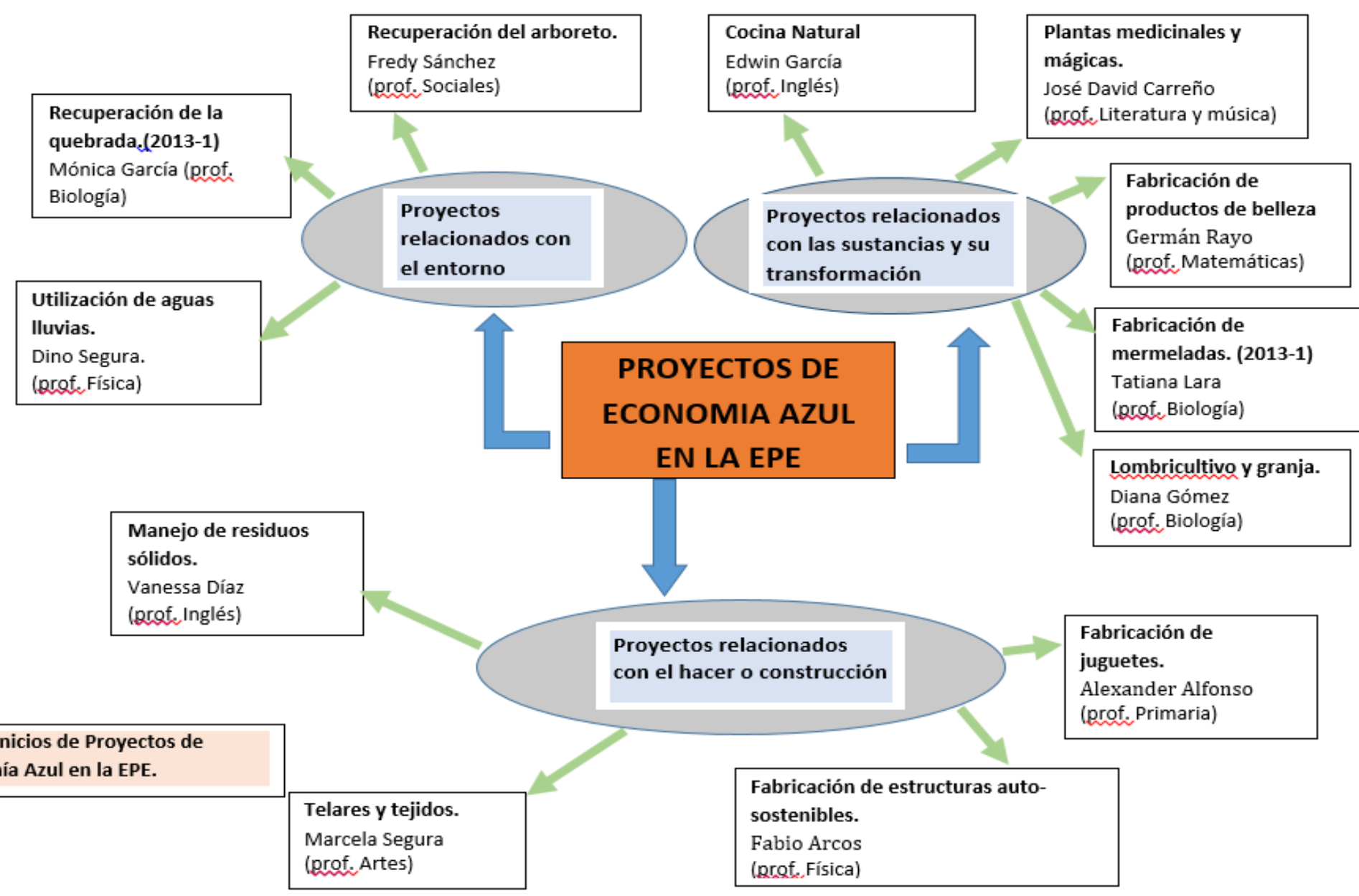

